\title{
A 3D-SHOCKWAVE VOLUME RENDERING ALGORITHM BASED ON FEATURE BOUNDARY DETECTION
}

\author{
Huawei Wang ${ }^{1,2}$, Liu $\mathrm{He}^{1}$, Zhiwei $\mathrm{Ai}^{1,2}$ and $\mathrm{Yi} \mathrm{Cao}^{1,2}$ \\ ${ }^{1}$ Laboratory of Computational Physics, Institute of Applied Physics and Computational Mathematics, Beijing 100088, \\ China \\ ${ }^{2}$ CAEP Software Center for High Performance Numerical Simulation, Beijing 100088, China
}

\begin{abstract}
The mechanism of shockwave propagation in complex environments plays an important role in the damage and protection assessment. At present, domain experts are realizing shockwave propagation numerical simulations with high scientific confidence on thousands of processing cores, and thus large-scale three-dimensional (3D) data fields containing fine physical features are output. It is desired to effectively reveal complex spatial structures of shockwaves in the data fields so as to facilitate domain experts to make in-depth observation and analysis. In this paper, a volume rendering algorithm based on feature boundary detection is proposed to visualize 3D shockwave data fields. In the algorithm, a two-dimensional (2D) boundary detection technique is generalized to the 3D case and further used to extract feature data containing the wavefronts, and then, the feature data are resampled and composited in the ray-casting manner. The algorithm has been preliminarily applied to 3D shock fields, and the results show that the algorithm can effectively analyze the shockwave features and clearly show the 3D spatial structures of the wavefronts.
\end{abstract}

\section{KEYWORDS}

Shockwave, Volume Rendering, Boundary Detection, Wavefront, Visualization

\section{INTRODUCTION}

Relevant researches show that the destructive effect of an explosion is mainly caused by shock wave. As one of the main killing factors, shock wave can kill or destroy the target at a long distance. The mechanism of shockwave propagation in complex environments plays an important role in the damage and protection assessment. With the development of high performance computing, domain experts are using thousands of processing cores to realize high-confidence numerical simulations for the shockwave propagation mechanism. By means of numerical simulation, they carefully explore the propagation, diffraction and reflection laws of the shock wave under diverse conditions, and as a result large-scale 3D shockwave data fields with fine features are output. It is desired to effectively show complex shockwave features in these 3D data fields so that domain experts can perform in-depth observation and analysis.

To visualize 3D shockwave data fields, we can use direct volume rendering so as to see their internal structures. The direct volume rendering method transforms the discrete data in the 3D space directly into a 2D image without generating intermediate geometric primitives. Compared with the surface rendering method, the direct volume rendering method has stronger expressiveness of the data, so it has been widely used in the field of scientific visualization. Based on direct volume rendering, the visualization results of 3D shockwave data fields may provide domain experts with intuitive and high-quality references for analysis.

By assigning optical attributes to the sampling points of the volume data with a transfer function, the volume rendering method relies on translucent fusion to simulate the real observation effect of the material structure. It can increase/decrease the opacity of a matter via the transfer function so as to highlight/weaken its internal structure; however, it is usually difficult to reveal complex structural features via the transfer function in volume rendering. In order to get good rendering effect, one has to take much time and effort to design a complex transfer function, which is often high-dimensional and thus has an enormous parameter space. 
In the propagation process of shock wave, the physical properties such as pressure and density have numerical mutations on and behind the wave front, thus the shockwave visualization belongs to a kind of drawing problem of 3D boundary surfaces with complex structures. However, because the data field across/along the wave front has a trans-scale change, it is difficult to describe the wave front by one or more scalars, e.g. the pressure and the magnitude of its gradient. Therefore, traditional volume rendering methods for simple material boundary are difficult to be applied to the rendering of shock wave.

In this paper, boundary detection is introduced to extract feature data instead of transfer function in volume rendering, so a volume rendering algorithm based on feature boundary detection is proposed to effectively render wavefront structures in shockwave data fields. In the algorithm, we use the boundary detection method to analyze the data fields and extract the data layer containing the wave fronts. Taking the $2 \mathrm{D}$ slices of the 3D data fields as the processing granularity, we transform the feature detection problem into the $2 \mathrm{D}$ case and thus realize the generalization of $2 \mathrm{D}$ boundary detection to $3 \mathrm{D}$. Coupling boundary detection with volume rendering, we can then render a clear visual effect of shock wave fronts.

\section{RELATED WORK}

In order to effectively show internal features in data fields, a variety of volume rendering algorithms are proposed, including ray casting (Palmer et al., 1997; Ma, 1999), cell projection (Ma and Crockett, 1997), shear warp (Sano et al., 1997) and splatting (Li et al., 1997). Because the main calculation of volume rendering is focused on two stages: sampling and compositing, the typical parallel implementation mode includes the two steps of data parallel and image parallel, and the I/O strategy, data organization and load balance are the important factors affecting the parallel performance. In the distributed-parallel visualization software VisIt (Childs et al., 2010), Childs et al. (2005) proposed a contract-based visualization pipeline, which can perform some optimization strategies according to the rendering parameters, such as I/O pre-filtering and load balance, thus the execution efficiency of the pipeline is improved. They further proposed another load balancing optimization named by the hybrid parallel mode (Childs et al., 2006). Later, Moloney et al. (2007) proposed a dynamic load balancing strategy, which can estimate the rendering cost per pixel and distribute the computing tasks balancedly. Although the sort-last mode is widely used in parallel volume rendering, Moloney et al. (2011) found that several parallel volume rendering algorithms can have better performance in the sort-first mode. On large-scale parallel computers, Howison et al. (2012) proposed a MPI+OpenMP hybrid parallel volume rendering algorithm, which makes full use of the shared memory on multi-core nodes to reduce memory consumption and data traffic and thus scales successfully to 216000 processing cores.

In order to enhance the display effect of volume rendering, one of the most effective ways is to highlight the structural details in the volume data, such as boundaries, contours and so on. There have been some methods to enhance the effect of boundary display, for example, Lum and Ma (2004) proposed lighting transfer functions using gradient aligned sampling to highlight the boundary interfaces between substances. Tenginakai et al. (2001) proposed a statistical method to locate the salient iso-surfaces at material boundaries based on central moments of data. Roettger et al. (2005) proposed to classify material boundaries by grouping the spatial connected regions in the two-dimensional histogram. Huang et al. (2003) extended the transfer function to 3D by adding spatial information and then used a region growing algorithm based on cost function to accurately reveal material boundaries. Wang et al. (2010) used the Gaussian mixture model and the elliptical transfer function in the $2 \mathrm{D}$ feature space so as to improve the accuracy of the boundary interface and the design efficiency of the transfer function. Caban and Rheingans (2008) proposed texture-based transfer functions for direct volume rendering in order to effectively distinguish some structures and features with the same intensity and gradient values. Tzeng et al. (2005) proposed a volume classification approach coupling machine learning and painting metaphor, which enables the user to better extract materials of interest in a much higher dimensional space.

Edge detection is a basic problem in image processing and computer vision. The purpose of edge detection is to identify the points with obvious brightness changes in digital images. The classical edge detection algorithms include first-derivative detectors (such as Roberts detector, Sobel detector and Prewitt detector), second-derivative detectors (e.g. Laplacian detector) and nonlinear detectors (e.g. Kirsch detector). Generally speaking, with the increase of the sensitivity to edge, the sensitivity to noise increases at the same 
time for an edge detector; however, if removing the noise by image smoothing, the edge detector will have bigger uncertainty in edge location. Canny (1986) analyzed the edge quality for the existing detectors and then proposed Canny detector, which uses the optimal edge detection factors to suppress the noise and produce relatively complete edges. Later, some scholars extended and improved Canny detector from different aspects, including (Bergholm, 1987) and (Rothwell et al., 1995). Jin (2011) also proposed an improved Canny algorithm based on median filtering, which has a higher signal-to-noise ratio and can extract the edges more accurately. Recently, Yagüe-Fabra et al. (2013) proposed an edge detection method based on a 3D Canny algorithm for extracting the surfaces of industrial parts with homogeneous materials.

\section{FEATURE DETECTION OF SHOCK WAVEFRONT}

For a 3D shockwave data field, we first take its 2D slices as the basic processing granularity. Here, the input data field is represented as a multi-block rectilinear mesh. Slicing the data field perpendicular to the three axes, we obtain a series of 2D rectilinear meshes, which can be regarded as images in a sense. Figure 1 (Left) shows a slice of the shockwave pressure field. We then use the Canny algorithm to detect the boundaries of wave fronts on these slices.

In detail, the detection steps of the Canny algorithm are as follows:

1) Firstly, as a preprocessing step, smooth the image by a Gaussian filter to remove the noise. In this paper, the $5 \times 5$ Gaussian filter core is used.

2) Calculate the gradients of the image intensity. In order to detect the edges in different directions, the Canny algorithm uses two directional filters to detect horizontal and vertical edges in the smoothed image.

3) In order to make the detection results cleaner, use non-maximum suppression to eliminate false edges and re-process the noise. Non-maximum suppression is to suppress the elements that are not the maximum, and it is also a form of local maximum search.

4) In order to eliminate the remaining false edges, filter out the edges with small gradient values while retaining the edges with high gradient values, which can be achieved by using double thresholds: high threshold and low threshold. However, high and low thresholds usually need to be determined by continuous attempts.

After the above four steps, strong edge pixels will be retained in the final result. However, it is difficult to distinguish weak edge pixels. We use the connection method of edge tracking to determine which weak edges can be added to the final result. In order to track the edge connection, we need to observe the weak edge pixels and their 8 neighborhood pixels. As long as its eight neighbors contain a strong edge pixel, the weak edge pixel can be identified as a preserved point and then connected to the final result. As a result, we complete the connection of the edge lines, which forms the final detection results.
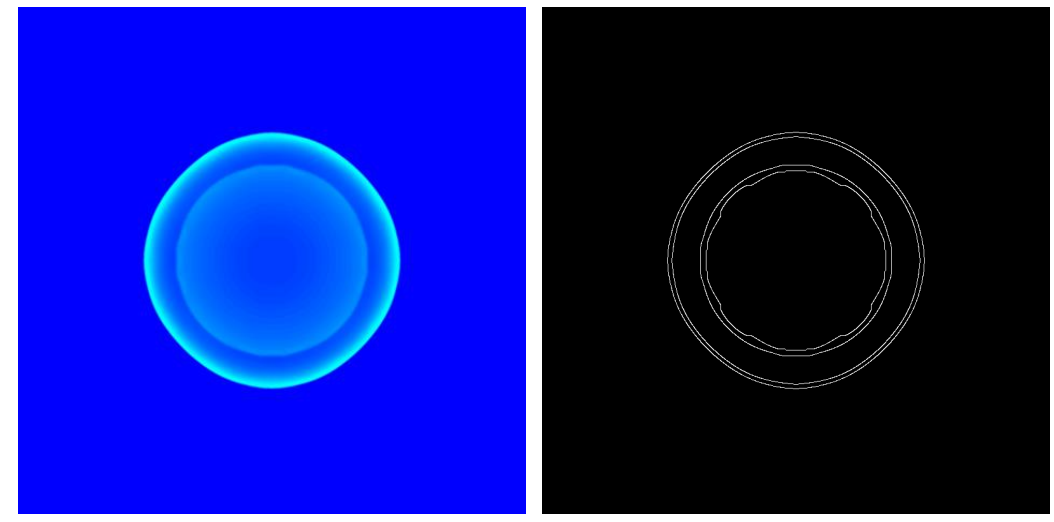

Figure 1. A shockwave data slice (Left) and the boundaries detected (Right)

Since the shockwave pressure takes a floating point value and varies violently in space, we need to carefully choose the parameters in the Canny algorithm. For example, in order to eliminate some unexpected boundaries in the wavefront detection, it is necessary to select appropriate high and low thresholds. In this paper, the low threshold is 0.016 , and the high threshold is 0.058 . Figure 1 (Right) shows the final detection 
results. The extracted boundaries have relatively good continuity, which proves the effectiveness of the Canny detection algorithm. However, there are two boundaries corresponding to each wave front, as shown in Figure 1 (Right).

To analyze the problem, we draw the variation chart of the underlying variable, as shown in Figure 2. Obviously, the variable value has a violent variation through each wave front. It is known in image processing that the image edge refers to the place where the pixel value of the image changes suddenly. In the variation chart in Figure 2, point 2 is the actual wavefront position; however, there is a mutation between point 1 and point 2 (namely front mutation), and there is also a mutation between point 2 and point 3 (namely back mutation), so two-layer boundaries appear for a wave front based on image edge detection.

One can see from Figure 2 that the front mutation is just a sudden pulse, which is much more violent than the back mutation, so the outer boundary corresponding to the front mutation is almost right on the wavefront while the inner one deviates from the wavefront obviously. We can observe the situation in Figure 3 . In Figure 3, we overlap the results of Canny boundary detection with the actual data slice, and it is shown that the outer boundary almost coincides with the actual shock wavefront. Therefore, for boundary detection results for each wave front, we keep only the outer ring and ignore the inner one so as to obtain a clearer resulting boundary.
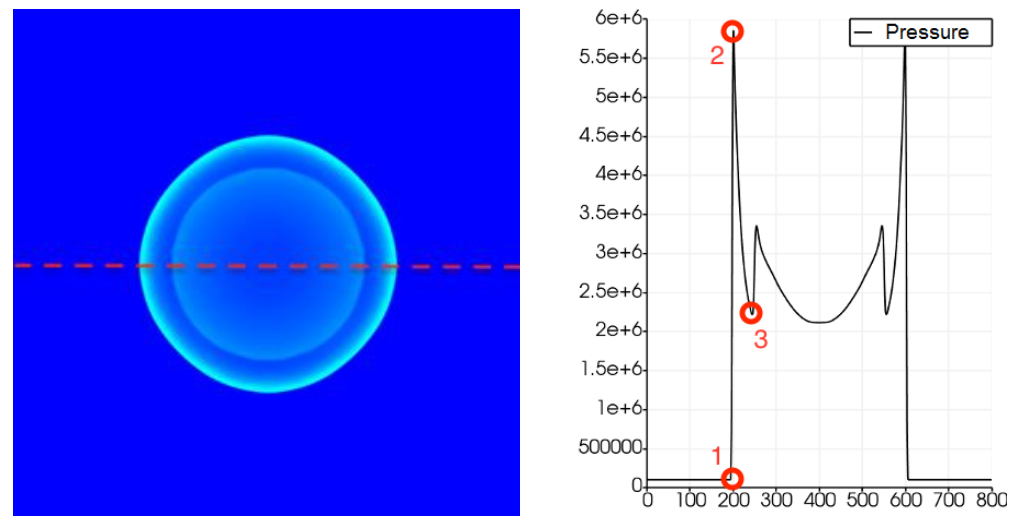

Figure 2. On a line (Left) the variation chart of the pressure is drawn (Right)

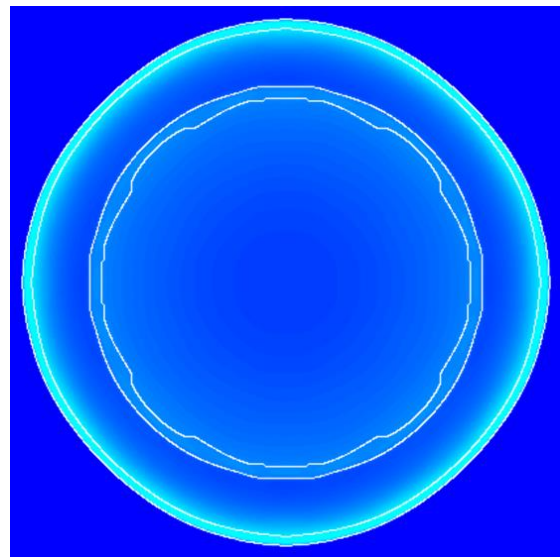

Figure 3. A shockwave slice overlapping the boundaries resulted by the Canny algorithm (amplified locally)

Suppose that the input 3D data field is based on an $m \times n \times k$ mesh. If slicing the data field perpendicular to an axis, e.g. the $\mathrm{Z}$ axis, we will have $k$ data slices, i.e. $k$ storeys of mesh cells. We then conduct the boundary detection on these slices using the Canny algorithm, where we will run into some different situations. For example, the boundary detection results may be as shown in Figure 4; at this time, we can also take the outer boundary and ignore the remaining ones for each wave front, similar to the situation in Figure 3 . For more special cases that are not easy to process, for example, some slices passing through the upper/bottom part of a shockwave sphere, we will then continue to perform the boundary detection perpendicular to $\mathrm{X}$ axis or further Y axis, until all boundaries of wave fronts are determined in the whole data field. 


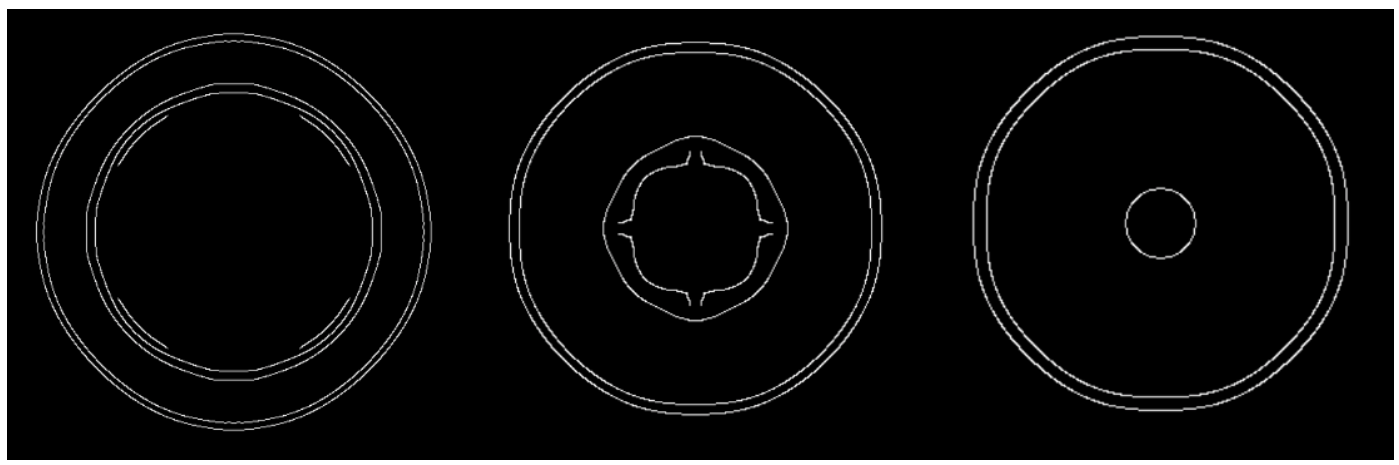

Figure 4. Some detection results on data slices using the Canny algorithm

After the wavefront boundary detection is performed for the whole data field, the cells around the boundaries will be taken as the feature data, and the rest areas are marked invalid. In detail, we take a little box surrounding each boundary point into the feature data, and the half width of the box is $d$ in three directions, e.g. $d=3$ in our experiments. That is to say, we thicken the boundaries, or give the feature data a thickness, so that they can be resampled along any rays without cracks during the process of volume rendering. Note that the wavefront feature data need to be extracted only once, and they will stay the same when the view angle changes interactively and accordingly the volume rendering pipeline is executed again.

\section{VOLUME RENDERING OF SHOCK WAVE}

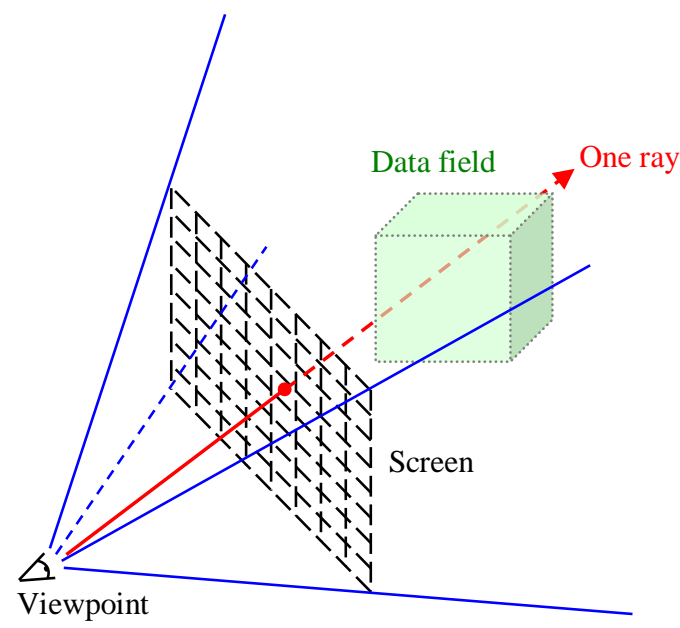

Figure 5. Ray-casting volume rendering

A simple description of ray-casting volume rendering is shown in Figure 5. A ray is cast from the viewpoint to each pixel in the screen, along which the data field is resampled. All sampling points will be mapped to color and opacity values from transfer functions. Compositing all sampling points on each ray according to the depth order, we then obtain the color for the pixel corresponding to the ray.

Coupling feature detection with ray casting, we then have our volume rendering algorithm for shockwave data fields as follows:

1) From a shockwave data field, extract wavefront feature data using the boundary detection method proposed in the previous section, and accordingly set the ghost zone tag to the original data field: 0 for all cells corresponding to the feature data and 1 otherwise;

2) In the rendering pipeline, neglecting the ghost zones, i.e. all cells whose ghost zone tags are equal to 1 , resample the original data field along each ray and meanwhile interpolate variable values for all sampling points; 
3) Composite the sampling points on each ray in the front-to-back order, and obtain the volume rendering image of wave front finally.

We have implemented the proposed volume rendering algorithm in the distributed-parallel visualization software VisIt. Because the wavefront detection method is a little complicated, its implementation is still serialized currently; we temporarily use an operator to merge the data field into one large block prior to wavefront detection. We will parallelize the whole algorithm in the next step to deal with large-scale data fields efficiently.

\section{EXPERIMENTAL RESULTS}
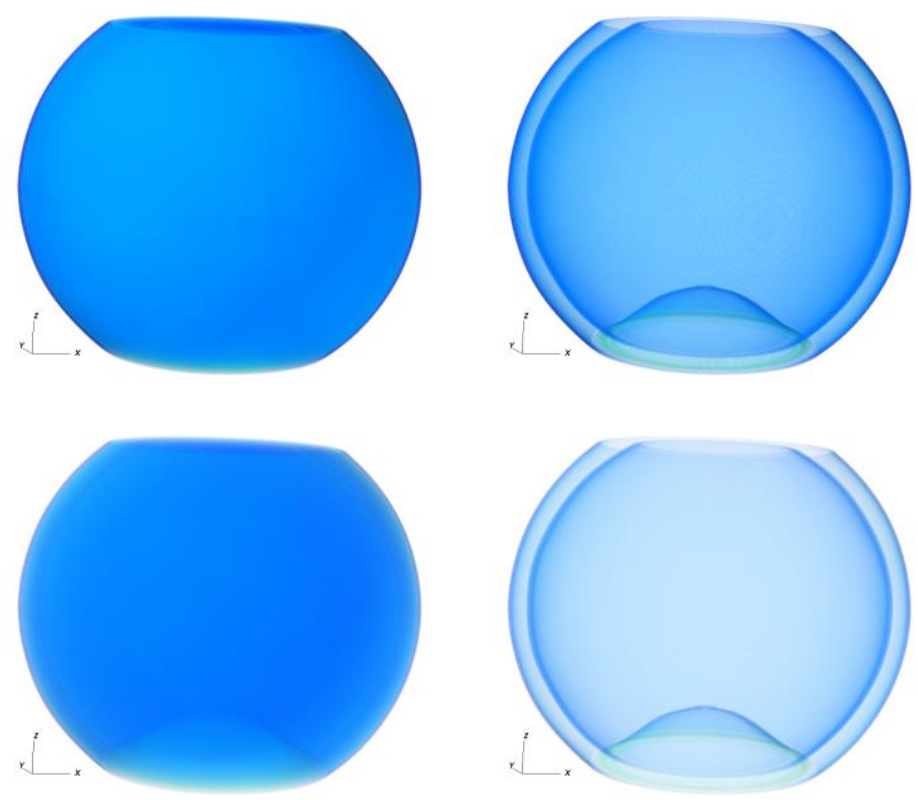

Figure 6. Comparisons of volume rendering results: The left column is for the traditional algorithm and the right column for the proposed algorithm; the opacity for the first row equals to 0.126 while the second row decreases to 0.042

For a test shockwave data field, we will check whether the proposed algorithm can clearly reveal the structure of shock wave front. The rendering results of shock wave using our algorithm are shown in Figure 6 (right). For comparison, we also render the data with the same parameters using the traditional ray-casting volume rendering algorithm, and the results are shown on the left of Figure 6.

At first, we set a small constant opacity of 0.126 (The maximum opacity is 1.0 in our program), as shown in the first row of Figure 6. For our algorithm, we can clearly see the structures behind and inside the front wavefront; but the internal structures are almost invisible for the traditional algorithm, although the opacity has been very small and the background field has been especially filtered out for the traditional algorithm. And then, further reducing the opacity to one third (i.e., the opacity becomes 0.042), we obtain the rendering results, as shown in the second row of Figure 6. The rendering effect for the traditional algorithm is still not ideal, and the internal wave fronts are only faintly seen.

In order to check the applicability, we further apply the algorithm to the time-varying process of shockwave propagation. The rendering results of wave fronts for the time-varying process are shown in Figure 7, where four typical time steps are listed, i.e. 0300, 0600, 0900 and 1200. The rendering results clearly show the evolution law of shockwave propagation: gradual expansion, obstacle reflection and further expansion. The results also show that the proposed algorithm can extract and render multi-wavefronts, even if they become irregular. 
Therefore, the algorithm proposed in this paper can be used to deal with 3D complex wavefront structures. The algorithm clearly shows the internal structures of wave fronts by extracting the wavefront feature data out of the data field and then rendering the feature data only. Because the rendering of wave front mainly relies on feature detection, there is no need to spend much time to interactively design a suitable opacity transfer function.

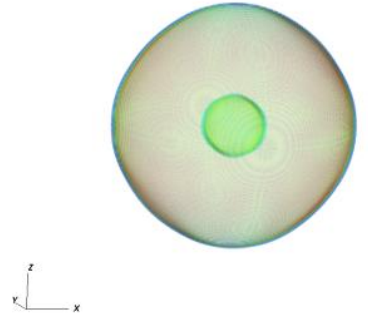

(a) Time step 0300

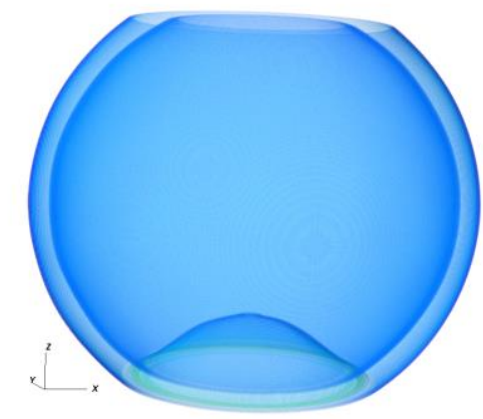

(c) Time step 0900

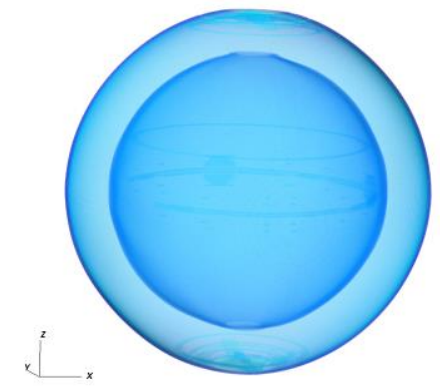

(b) Time step 0600

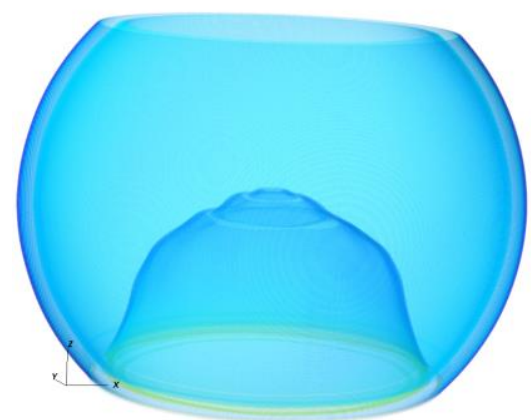

(d) Time step 1200

Figure 7 Time-varying wavefront evolutions in shockwave propagation

\section{CONCLUSION}

Aiming at numerical simulations of 3D shockwave, this paper presents a volume rendering algorithm based on feature boundary detection for extracting and exhibiting wavefront spatial structures from violently-varying 3D shockwave data fields. In order to detect 3D wave fronts, the algorithm generalizes the boundary detection technique for $2 \mathrm{D}$ image to $3 \mathrm{D}$ volume data, and accordingly the feature data containing wave fronts are extracted from shockwave data fields. And then, the algorithm resamples and fuses the feature data according to the ray casting method, thus the clear visualization of complex wavefront structures is realized in the end. The algorithm characterizes the physical feature of wave front by the extracted feature data instead of a transfer function, thus there is no need to take much time and effort to design the transfer function. The algorithm has been initially applied to the visualization of 3D shockwave data fields, supporting domain experts to observe and analyze the evolution process of shock wave.

In the next step, we will continue to improve the proposed algorithm. Firstly, we will improve the 3D boundary detection technique to well adapt to complex wavefronts as well as general situations. And then, we will enhance the illumination effect on wave fronts to make them more prominent from the volume rendering image. In addition, we will further address the problem of efficiently-parallel implementation so as to deal with large-scale data fields. As a result, the high quality visualization of large-scale complex shockwave data fields will be achieved. 


\section{ACKNOWLEDGEMENT}

This work was supported by National Key R\&D Program of China (No. 2017YFB0202203) and Foundation of Laboratory of Computational Physics.

\section{REFERENCES}

Bergholm, F., 1987. Edge focusing. In IEEE Transactions on Pattern Analysis and Machine Intelligence, Vol. 9, No. 6, pp. 726-741.

Caban, J. and Rheingans, P., 2008. Texture-based Transfer Functions for Direct Volume Rendering. In IEEE Transactions on Visualization and Computer Graphics, Vol. 14, No. 6, pp. 1364-1371.

Canny, J., 1986. A computational approach to edge detection. In IEEE Transactions on Pattern Analysis and Machine Intelligence, Vol. 8, No. 6, pp. 679-698.

Childs, H. et al., 2005. A contract based system for large data visualization. Proceedings of IEEE Visualization '05. Minneapolis, MN USA, pp. 191-198.

Childs, H. et al., 2006. A scalable, hybrid scheme for volume rendering massive data sets. Proceedings of EGPGV '06. Braga, Portugal, DOI:10.2312/EGPGV/EGPGV06/153-161.

Childs, H. et al., 2010. Extreme scaling of production visualization software on diverse architectures. In IEEE Computer Graphics and Applications, Vol. 30, No. 3, pp. 22-31.

Howison, M. et al., 2012. Hybrid parallelism for volume rendering on large, multi- and many-core systems. In IEEE Transactions on Visualization and Computer Graphics, Vol. 18, No. 1, pp. 17-29.

Huang, R. et al., 2003. Visualizing industrial CT volume data for nondestructive testing applications. Proceedings of IEEE Visualization '03. Seattle, WA USA, DOI:10.1109/VISUAL.2003.1250418.

Jin, Y., 2011. Research on an Improved Canny Algorithm for Image Edge Detection. Master's thesis, Chongqing Normal University. [In Chinese]

Li, P. et al., 1997. ParVox-a parallel volume rendering system for distributed visualization. Proceedings of Symposium on Parallel Rendering '97. Phoenix, AZ USA, pp. 7-14.

Lum, E. and Ma, K., 2004. Lighting transfer functions using gradient aligned sampling. Proceedings of IEEE Visualization '04. Austin, TX USA, pp. 289-296.

Ma, K. and Crockett, T., 1997. A scalable parallel cell-projection volume rendering algorithm for three dimensional unstructured data. Proceedings of Symposium on Parallel Rendering '97. Phoenix, AZ USA, pp. 95-104.

Ma, K., 1999. Parallel rendering of 3D AMR data on the SGI/Cray T3E. Proceedings of the 7th Symposium on the Frontiers of Massively Parallel Computation. Annapolis, MD USA, pp. 138-145.

Moloney, B. et al., 2007. Scalable sort-first parallel direct volume rendering with dynamic load balancing. Proceedings of EGPGV '07. Lugano, Switzerland, DOI:10.2312/EGPGV/EGPGV07/045-052.

Moloney, B. et al., 2011. Sort-first parallel volume rendering. In IEEE Transactions on Visualization and Computer Graphics, Vol. 17, No. 8, pp. 1164-1177.

Palmer, M. et al., 1997. Exploiting deep parallel memory hierarchies for ray casting volume rendering. Proceedings of Symposium on Parallel Rendering '97. Phoenix, AZ USA, pp. 15-22.

Roettger, S. et al., 2005. Spatialized transfer functions. Proceedings of EuroVis05 Joint Eurographics - IEEE VGTC Symposium on Visualization. Leeds, United Kingdom, DOI:10.2312/VisSym/EuroVis05/271-278.

Rothwell, C. et al., 1995. Driving vision by topology. Proceedings of IEEE International Symposium on Computer Vision (ISCV '95). Coral Gables, FL USA, pp. 395-400.

Sano, K. et al., 1997. Parallel processing of the shear-warp factorization with the binary-swap method on a distributed-memory multiprocessor system. Proceedings of Symposium on Parallel Rendering '97. Phoenix, AZ USA, pp. 87-94.

Tenginakai, S. et al., 2001. Salient iso-surface detection with model-independent statistical signatures. Proceedings of IEEE Visualization '2001. San Diego, CA, USA, DOI:10.1109/VISUAL.2001.964516.

Tzeng, F. et al., 2005. An intelligent system approach to higher-dimensional classification of volume data. In IEEE Transactions on Visualization and Computer Graphics, Vol. 11, No. 3, pp. 273-284.

Wang, Y. et al., 2010. Volume exploration using ellipsoidal Gaussian transfer functions. Proceedings of 2010 IEEE Pacific Visualization Symposium (PacificVis). DOI:10.1109/PACIFICVIS.2010.5429612.

Yagüe-Fabra, J. et al., 2013. A 3D edge detection technique for surface extraction in computed tomography for dimensional metrology applications. In CIRP Annals - Manufacturing Technology, Vol. 62, No. 1, pp. 531-534. 